\title{
Brain metastases from lung cancer with neuropsychiatric symptoms as the first symptoms
}

\author{
Kai Dong ${ }^{1}$, Lei Liu ${ }^{2}$, Zhipeng Yu ${ }^{1}$, Di Wu ${ }^{3}$, Qian Zhang ${ }^{1}$, Xiaoqin Huang ${ }^{1}$, Jianping Ding ${ }^{1}$, Haiqing Song ${ }^{1}$ \\ ${ }^{1}$ Department of Neurology, ${ }^{2}$ Department of Thoracic Surgery, ${ }^{3}$ China-America Institute of Neuroscience, Xuanwu Hospital, Capital Medical \\ University, Beijing 100053, China \\ Contributions: (I) Conception and design: K Dong, H Song; (II) Administrative support: L Liu, Z Yu; (III) Provision of study materials or patients: D \\ Wu; (IV) Collection and assembly of data: Q Zhang; (V) Data analysis and interpretation: K Dong, H Song; (VI) Manuscript writing: All authors; (VII) \\ Final approval of manuscript: All authors. \\ Correspondence to: Haiqing Song, MD. Department of Neurology, Xuanwu Hospital, Capital Medical University, Beijing 100053, China. \\ Email: songhaiqing@xwh.ccmu.edu.cn.
}

Background: To investigate the neuropsychiatric symptoms and their treatment and outcomes in lung cancer patients with brain metastases $(\mathrm{BM})$, with an attempt to achieve early detection and prompt management of these symptoms.

Methods: Ten lung cancer patients ( 8 males and 2 females) with BMs who were treated in our center from 2013 to 2019 were enrolled in this analysis. Without exception, all 10 patients presented with chief complaints of neuropsychiatric symptoms, and BMs were eventually diagnosed. Appropriate treatments were offered, and all patients were followed up.

Results: Two patients died (case 5 died of sudden massive hemoptysis, and case 6 died after his families refused to receive the invasive treatment). Data on 3- and 5-year survival have been obtained from one patient each. The average follow-up duration was 19.4 months (except that two patients were hospitalized only once, and one patient received the second follow-up visit only 9 days after the first visit).

Conclusions: The possibility of BM from lung cancer should be considered when a lung cancer patient develops neuropsychiatric symptoms, and timely diagnosis treatment should be arranged accordingly.

Keywords: Lung cancer; brain metastases (BM); neuropsychiatric symptoms

Submitted Jul 05, 2019. Accepted for publication Oct 11, 2019.

doi: $10.21037 /$ tlcr.2019.10.02

View this article at: http://dx.doi.org/10.21037/tlcr.2019.10.02

\section{Introduction}

Lung cancer is one of the most common malignant tumors worldwide. It can easily spread to the brain, bone, liver, lymph, and other organs/tissues. The disease condition rapidly worsens after metastasis, and the patient's survival is drastically shortened. The incidence of brain metastases (BM) is remarkably higher in patients with lung cancer patients than in those with melanoma, breast cancer, kidney cancer, colorectal cancer, and other common tumors (1). The most common primary tumors that spread to the brain were non-small cell lung cancer (NSCLC) and small cell lung cancer (SCLC) (34.5\% and $21.4 \%$, respectively) (2). In our current study, we elucidated the diagnosis, treatment, and follow-up of 10 lung cancer patients with BM who presented with neuropsychiatric symptoms (Table 1). Most patients with lung cancer are in the poor physical condition and often can not find out whether their body undergoes certain changes. When patients have discomfort in other parts of the body, they are rarely aware of the possibility of lung cancer metastasis. Rather, these problems are often considered to be the accompanying symptoms of lung cancer, so are neuropsychiatric symptoms. Therefore, few lung cancer patients undergo further examinations due to neuropsychiatric symptoms. Furthermore, abnormal gait (walking), dizziness, fatigue, and headache are common 


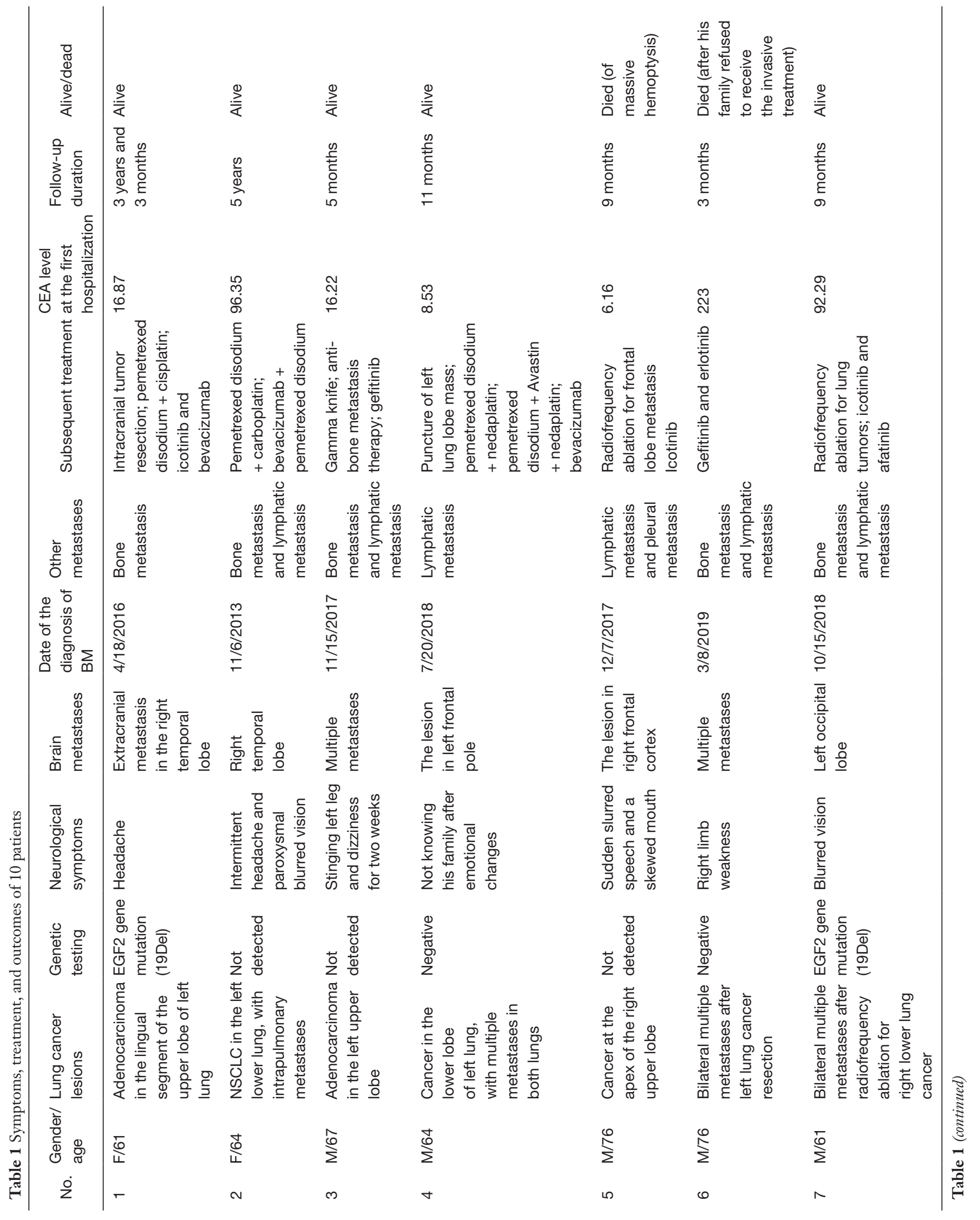




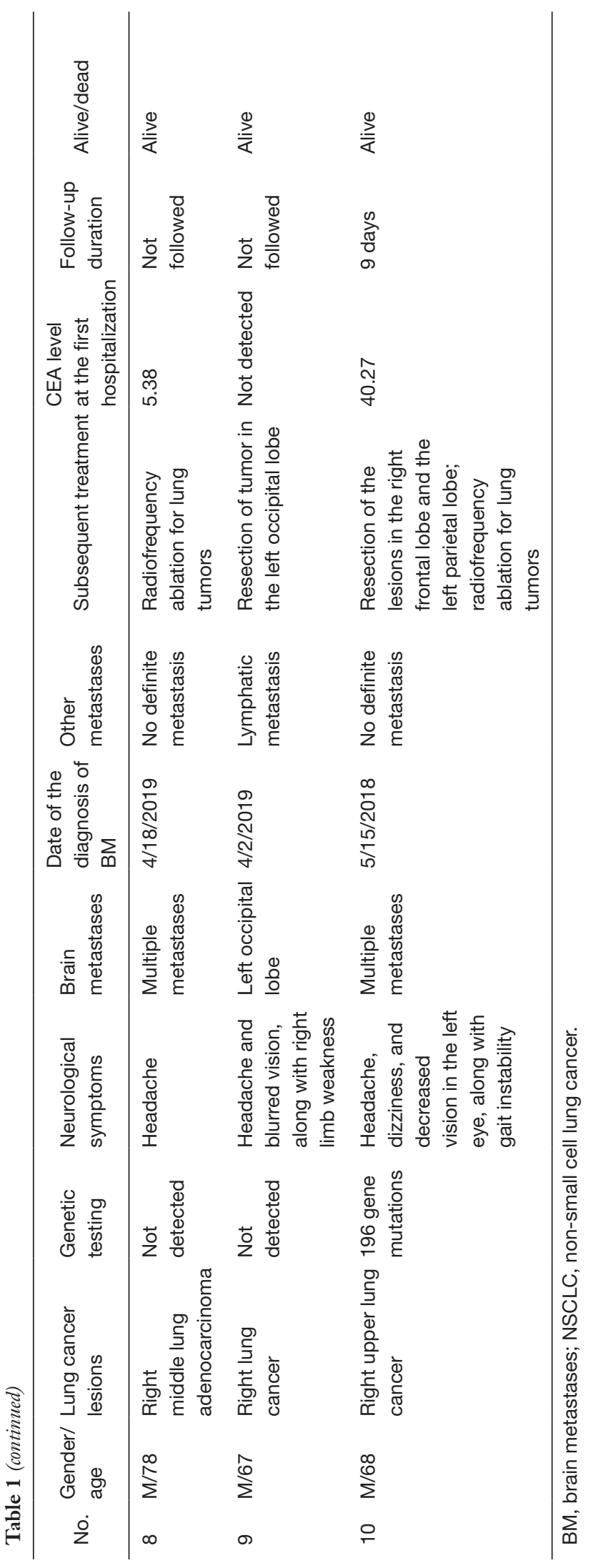

symptoms/signs in non-cancer patients and thus are often not suggestive of BM. As a result, early diagnosis of BM from lung cancer is difficult. The conditions are often worsened in most cases when lung cancer patients present with neuropsychiatric symptoms. When a lung cancer patient present with neuropsychiatric symptoms, the possibility of BM from lung cancer should be considered immediately. An early diagnosis will buy valuable time for treatment. Although the duration of neuropsychiatric symptoms differed among these 10 patients described in this article, BM from lung cancer was eventually confirmed in all patients.

\section{Methods}

\section{Case 1}

A 61-year-old female patient with adenocarcinoma in the lingual segment of the upper lobe of the left lung was admitted due to "headache for 4 months and right temporal mass for more than 2 months". There was no history of special diseases. After admission, she was diagnosed as extracranial metastasis in the right temporal lobe from lung cancer. The diagnosis of BM was made on April 18, 2016. The interval from the onset of neuropsychiatric symptoms to the diagnosis of BM was about 4 months. Genetic testing in this patient showed an EGFR exon 19 deletions (19-Del). Bone metastasis was also detected. A series of treatments, including surgical treatment (intracranial tumor resection), chemotherapy (pemetrexed disodium + cisplatin), targeted treatment (icotinib and bevacizumab), and symptomatic treatment, were given. The patient has been hospitalized 18 times in our hospital so far and is still alive.

\section{Case 2}

A 64-year-old female patient with left lower NSCLS with intrapulmonary metastasis was admitted due to "intermittent headache for more than 1 month and paroxysmal blurred vision for 2 weeks". The patient had a history of hypertension. After admission, she was diagnosed as metastasis in the right temporal lobe from lung cancer. The diagnosis of BM was made on November 6,2013 . The interval from the onset of neuropsychiatric symptoms to the diagnosis of BM was 1 month. No genetic testing was performed in this patient. The patient also had bone metastasis and lymphatic metastasis. A series of treatments, including chemotherapy (pemetrexed disodium 
+ carboplatin), targeted treatment (bevacizumab), and symptomatic treatment, were given. The outcome of the brain lesion can be seen in Figure 1. The patient has been hospitalized 19 times in our hospital so far and is still alive.

\section{Case 3}

A 67-year-old male patient with adenocarcinoma of the left upper lobe was admitted due to "tingling in the left leg for 2 months and dizziness for 2 weeks". He had previously undergone tracheoscopy and supraclavicular lymph node biopsy. After admission, he was diagnosed as multiple BMs from lung cancer. The diagnosis was made on November 15,2017 . The interval from the onset of neuropsychiatric symptoms to the diagnosis of BM was 2 months. No genetic testing was performed in this patient. The patient also had bone metastasis and lymphatic metastasis. A series of treatments, including surgical treatment (gamma knife surgery for brain metastasis), targeted therapy (gefitinib), treatment for bone metastasis, and the symptomatic treatment, were applied. The outcome of the brain lesion can be seen in Figure 2. The patient has been hospitalized 6 times in our hospital and is still alive.

\section{Case 4}

A 64-year-old male patient with left lower lobe cancer with multiple lung metastases was admitted to our hospital due to "not knowing his family for 1 week after emotional changes". His previous disease histories included hypertension, diabetes, coronary heart disease, and cerebral infarction (twice). After admission, it was found that the lung cancer had spread to the left frontal pole. The diagnosis was made on July 20, 2018. The interval from the onset of neuropsychiatric symptoms to the diagnosis of $\mathrm{BM}$ was 1 week. Genetic testing showed a negative result. In addition, the patient also had lymphatic metastasis. A series of treatments including puncture of left lung lobe mass, chemotherapy (pemetrexed disodium + nedaplatin; or pemetrexed disodium + Avastin + nedaplatin), targeted therapy (bevacizumab), and symptomatic treatment were offered. The patient has been hospitalized 13 times in our hospital and is still alive.

\section{Case 5}

A 76-year-old male patient with cancer at the apex of the right upper lobe was admitted due to "sudden slurred speech and a skewed mouth for 3 days". His disease histories included: hyperlipidemia; carotid atherosclerosis for 40 years; smoking for 60 years; right pneumothorax for 20 years; ligation for right bullae of the lung; and ligation for left bullae of the lung due to the left pneumothorax three years ago. After admission, he was diagnosed as metastasis beneath the right frontal cortex from lung cancer. The diagnosis was made on December 7, 2017. The interval from the onset of neuropsychiatric symptoms to the diagnosis of BM was only 3 days. No genetic testing was performed in this patient. In addition, the patient also had lymphatic metastasis and pleural metastasis. A series of treatments, including radiofrequency ablation for frontal lobe metastasis, targeted treatment (icotinib), and symptomatic treatment was offered. The patient was hospitalized 4 times in our hospital and died of massive hemoptysis during the last hospitalization on August 30, 2018.

\section{Case 6}

A 76-year-old male patient with multiple bilateral metastases after left lung cancer resection was admitted due to "right limb weakness for 3 days". His histories included: (I) Lesions were found in the left lung 9 years ago during a health check-up. He underwent left-sided thoracoscopic lung cancer resection in Beijing People's Hospital (with unknown pathological results); (II) hypertension for 8 years; and (III) prostate hyperplasia for more than 8 years. After admission, he was diagnosed as multiple BMs from lung cancer. The diagnosis was made on March 8, 2019. The interval from the onset of neuropsychiatric symptoms to the diagnosis of BM was only 3 days. Genetic testing showed negative results. The patient also had bone metastasis and lymphatic metastasis. A series of treatments, including targeted therapy (gefitinib and erlotinib) and symptomatic treatment, were offered. The patient was hospitalized 5 times in our hospital and died on June 9, 2019, after his family refused to receive invasive treatment.

\section{Case 7}

A 61-year-old male patient with multiple bilateral metastases after radiofrequency ablation for right lower lung cancer was admitted due to "blurred vision for 1 day". His previous histories included gallbladder stones, pneumothorax, and CT-guided local destruction of lung tumors. After admission, he was diagnosed as metastasis in the left occipital lobe from lung cancer. The diagnosis was 

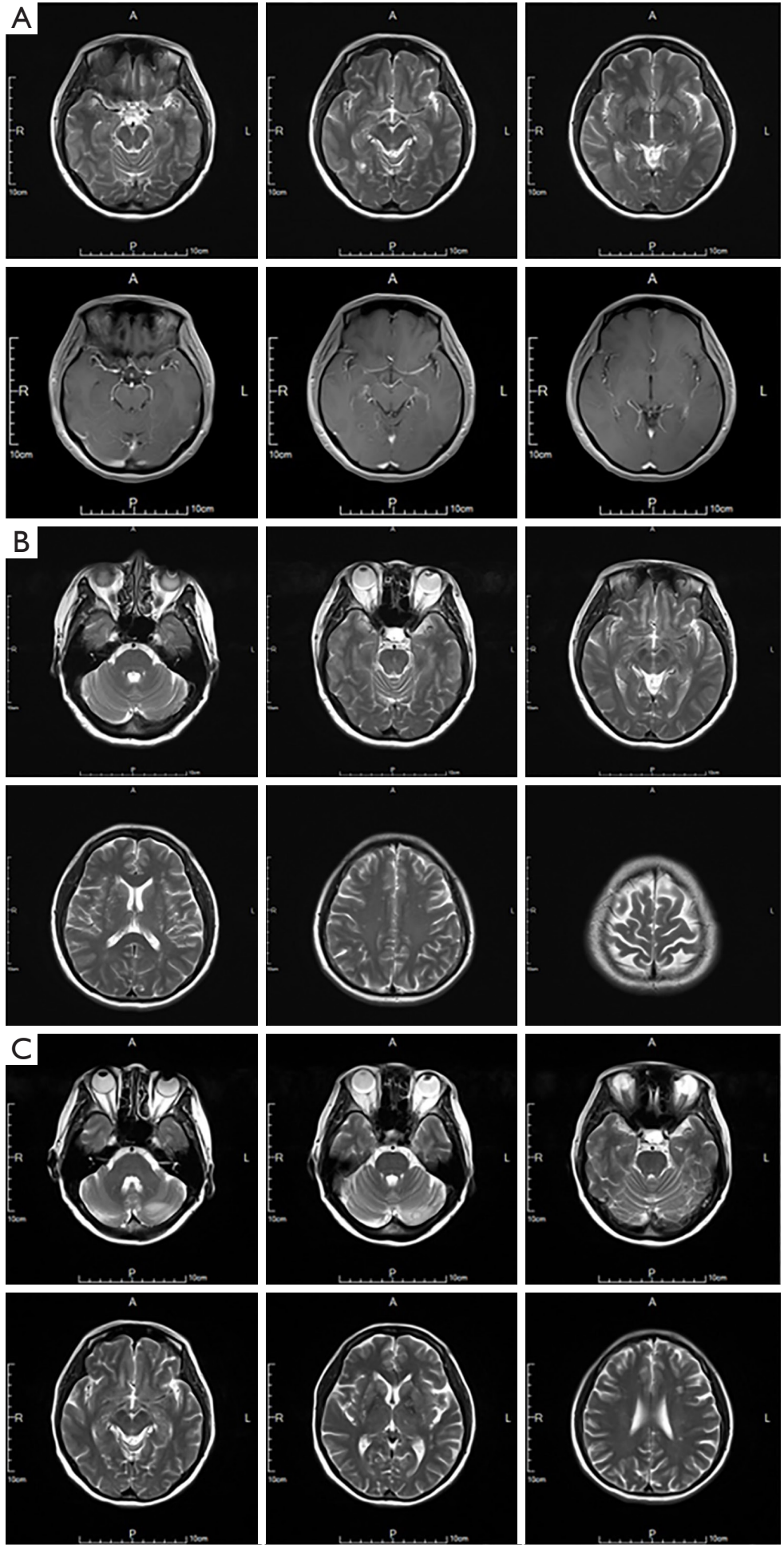

Figure 1 (A) MRI on November 6, 2013 showed metastasis in right temporal lobe, along with white matter degeneration. (B) After treatment with pemetrexed disodium + carboplatin, MRI on April 5, 2016 did not detect any newly-onset lesion in brain. Multiple lacunar infarction and ischemic foci were visible in the brain, along with white matter degeneration. (C) The chemotherapy with pemetrexed disodium and targeted therapy with bevacizumab continued. MRI on August 6, 2018 revealed new lesions in left cerebellar hemisphere, suggesting the possible metastatic tumors. Multiple lacunar infarction and ischemic foci, along with mild white matter degeneration. 

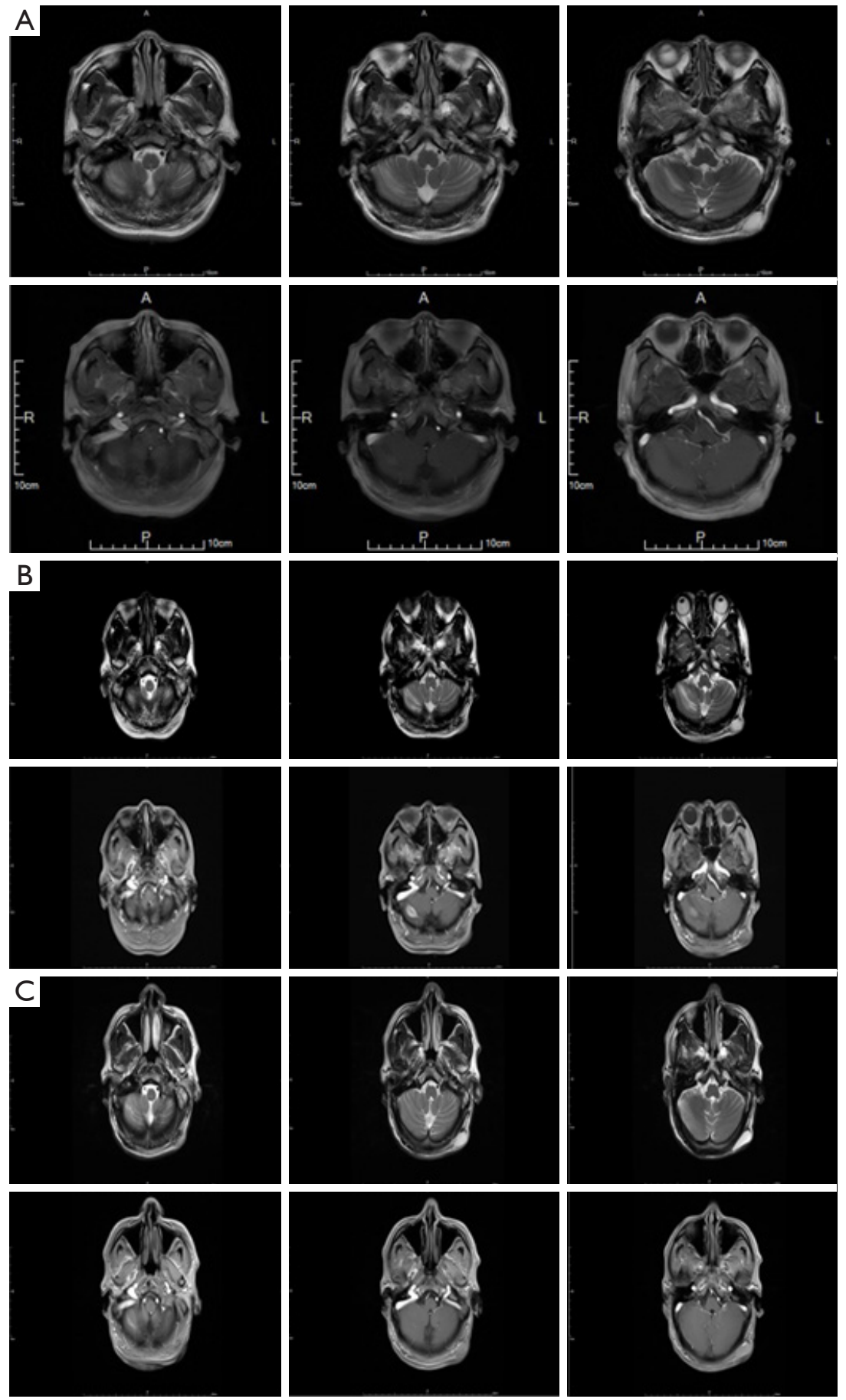

Figure 2 (A) MRI on November 15, 2017, revealed that the abnormal signals in the right cerebellar hemisphere were obviously enhanced, suggesting the possibility of metastasis. The abnormal meningeal signals in right midline were suggestive of meningioma. As the metastases were not ruled out, the abnormal signals in right frontal and left occipital lobes were suggestive of lipoma. (B) After symptomatic treatment such as analgesia, MRI on December 26, 2017 showed enlarged meningeal lesions in the right cerebellar hemisphere and the right midline, which were suggestive of multiple metastases. Abnormal enhancement at the fifth cervical vertebral body was detected, and the possibility of metastasis was considered. Abnormal signals in right frontal lobe and left occipital lobe were suggestive of lipoma. (C) After chemotherapy with gefitinib, gamma knife, and symptomatic treatment (e.g., analgesia), MRI on March 30, 2018 showed that the lesions in right cerebellar hemisphere significantly shrank and the dural lesions at right midline disappeared; lipoma might be present in the right frontal lobe and in the left occipital lobe. 
made on October 15, 2018. The interval from the onset of neuropsychiatric symptoms to the diagnosis of BM was only 1 day. Genetic testing showed the EGF2 gene mutation (19Del). The patient also had bone metastasis and lymphatic metastasis. A series of treatments, including radiofrequency ablation for lung cancer, targeted treatment (icotinib and afatinib), and symptomatic treatment was offered. The outcome of the brain lesion can be seen in Figure 3. The patient has been hospitalized 7 times in our hospital and is still alive.

\section{Case 8}

A 78-year-old male patient with right middle lung adenocarcinoma was admitted due to "headache for 4 months and right temporal mass for more than 2 months". He had a 4-year history of cerebral infarction. After admission, he was diagnosed as multiple BMs from lung cancer. The diagnosis of BM was made on April 18, 2019. The interval from the onset of neuropsychiatric symptoms to the diagnosis of $\mathrm{BM}$ was 1 month. No genetic testing was performed in this patient. No definite metastasis was found in other sites. CT-guided radiofrequency ablation of lung tumors and appropriate symptomatic treatment were given to the patient. This patient was hospitalized once in our hospital. Currently, his disease condition is fair.

\section{Case 9}

A 67-year-old male patient with right lung cancer was admitted due to "headache and blurred vision for six months and right limb weakness for half a month". He received an inguinal hernia repair 19 years ago. After admission, he was diagnosed as metastasis in the left occipital lobe from lung cancer. The diagnosis was made on April 2, 2019. The interval from the onset of neuropsychiatric symptoms to the diagnosis of BM was 6 months. No genetic testing was performed in this patient. In addition, the patient also had lymphatic metastasis. After resection of the tumor in the left occipital lobe and symptomatic treatment, the postoperative disease condition was fair. This patient was hospitalized once in our hospital.

\section{Case 10}

A 68-year-old male patient with cancer of the right upper lobe was admitted due to "headache, dizziness, and decreased vision in left eye for 2 months and gait instability for 1 month". He had a history of prostatic hyperplasia. After admission, he was diagnosed as multiple BMs from lung cancer. The diagnosis was made on May 15, 2018. The interval from the onset of neuropsychiatric symptoms to the diagnosis of BM was 2 months. Genetic testing showed 196 gene mutations. No definite metastasis was found in other sites. The lesions in the right frontal lobe and the left parietal lobe were resected, followed by CT-guided radiofrequency ablation of lung tumors. The patient is still alive. This patient has been hospitalized twice in our hospital.

\section{Discussion}

BMs from lung cancer are often fatal and thus drastically lower the survival rate of lung cancer patients (3). Lung cancer can easily spread to the brain and is the most common primary tumor for BM (4-6). Patients with BM from lung cancer usually have a poor quality of life and unfavorable prognosis. The average expected overall survival (OS) was only 4-7 months without local treatment, such as neurosurgery or radiosurgery (7). Thus, a prompt and accurate diagnosis of BM from lung cancer is particularly important. Villano et al. compared the incidence of BMs from various tumors and found the highest incidence of $\mathrm{BM}$ was from lung cancer (8).

In our current study, we analyzed 10 cases of BMs from lung cancer. The chief complaints were neuropsychiatric symptoms in each patient, suggesting the onset of the neuropsychiatric symptoms might indicate the metastasis of lung cancer. Therefore, the possibility of BM should be alerted when a lung cancer patient presents with neuropsychiatric symptoms. However, some neuropsychiatric symptoms are neglected in many lung cancer patients due to poor physical condition. These symptoms may be common and can be seen in many normal people. Many times the symptoms are ignored by patients and their families, resulting in a long interval between the development of BM and the hospital visit. Therefore, the lung cancer patients and their families should be routinely informed about the neuropsychiatric symptoms; once symptoms such as dizziness, headache, abnormal gait (walking), and limb weakness occur, the patient should timely come to the hospital for examinations. Second, lung cancer patients should take regular follow-up examinations (9), during which cranial imaging should be performed routinely. Patients with BMs often have metastases in other organs/tissues, such as bone and lymphatic tissue. Thus, the 

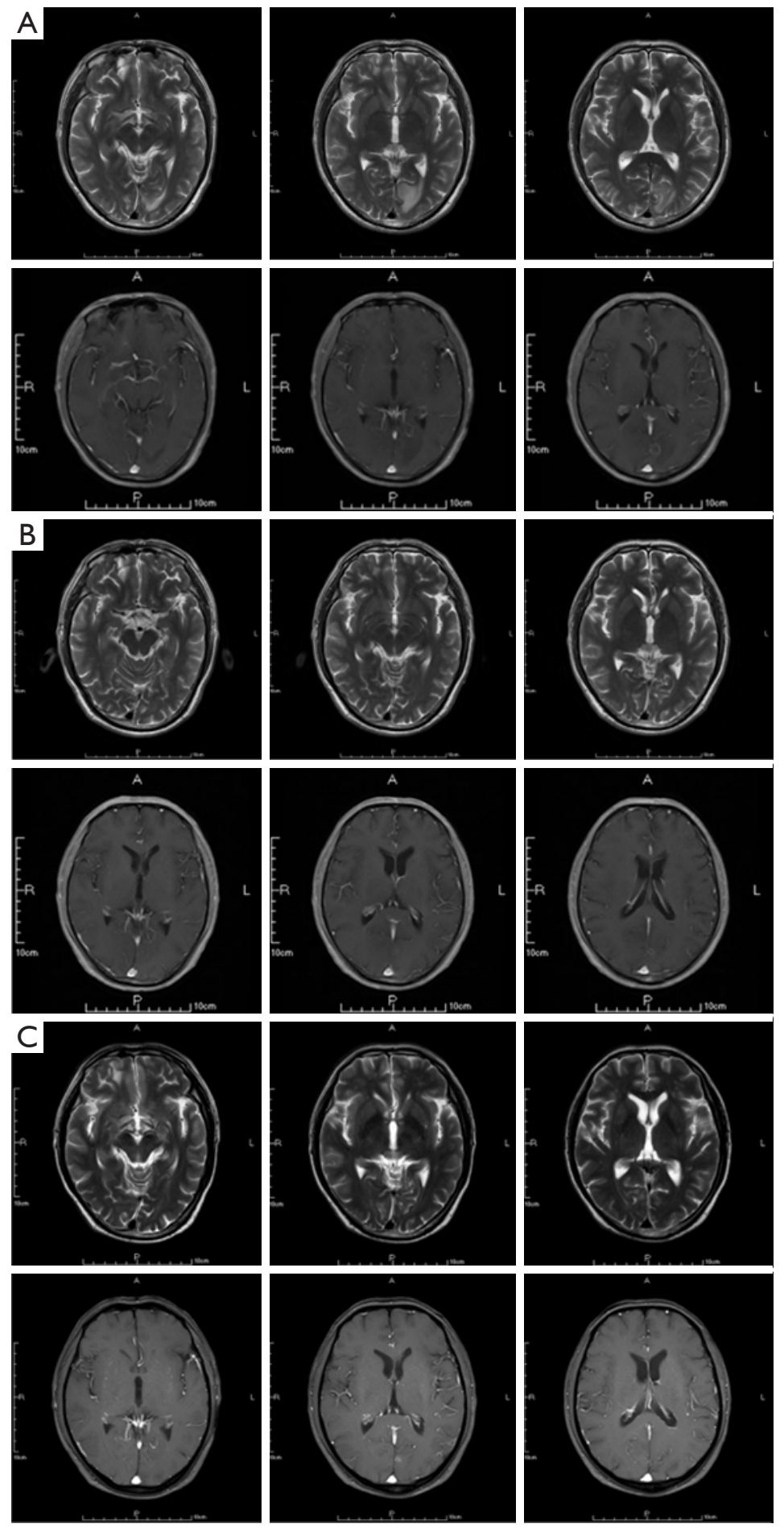

Figure 3 (A) MRI on October 15, 2018, showed abnormal signals in left occipital lobe, suggesting the possibility of metastasis; the softened lesions were seen in right frontal pole, along with multiple ischemic foci in the brain. (B) After CT-guided local destruction of lung tumor, MRI on October 24, 2018 showed that the left occipital metastases were significantly smaller than before; the softened lesions were seen in right frontal pole, along with multiple ischemic foci in the brain. (C) After targeted therapy with alectinib hydrochloride, MRI on May 13, 2019 showed that the left occipital metastases were similar, along with a few high signals, which were suggestive of hemorrhagic foci. The softened lesions were seen in right frontal pole, along with multiple ischemic foci in the brain. 
presence of these metastases is highly suggestive of BMs (10). In addition, Ainsworth et al. investigated the application of quantitative and texture analyses of magnetization transfer imaging in the early diagnosis of BMs (11). Only in this way can we achieve the early detection and early treatment of BMs from lung cancer.

Prompt screening and early diagnosis and treatment of BMs from lung cancer may increase the survival rate. The currently available treatments include surgery, targeted therapy, and radiochemotherapy (12-15). Zustovich et al. explored the preliminary outcomes of patients with BMs from non-small cell lung cancer treated with bevacizumabbased therapies. Thirteen patients ( 9 males and 4 females) had a median progression-free survival (PFS) of 9.1 months (range: 0.9-39.2 months) and median OS of 9.6 months (range: 3-41.5 months) (16). Whole-brain radiation therapy (WBRT) is considered the standard treatment (17-19). For any micrometastasis undetectable by brain imaging, WBRT can prevent any intracranial recurrence and lower the risk of death caused by neurological problems (19). In our current study, two patients died (Case 5 died of sudden massive hemoptysis, and Case 6 died after his families refused to receive the invasive treatment). Data on 3-and 5 -year survival have been obtained from one patient each.

\section{Conclusions}

$\mathrm{BM}$ is a common cause of neurological symptoms in patients with lung cancer. When patients with lung cancer are found to have neurological symptoms, the possibility of BM should be considered. Early diagnosis and treatment will help to relieve symptoms and prolong survival. Multidisciplinary treatments, including surgery, radiochemotherapy, targeted therapy, and symptomatic treatment, should be offered to patients with BM from lung cancer.

\section{Acknowledgments}

None.

\section{Footnote}

Conflicts of Interest: The authors have no conflicts of interest to declare.

Ethical Statement: The authors are accountable for all aspects of the work in ensuring that questions related to the accuracy or integrity of any part of the work are appropriately investigated and resolved. The written informed consent was obtained from all patients.

\section{References}

1. Nieder C, Haukland E, Mannsåker B, et al. Presence of Brain Metastases at Initial Diagnosis of Cancer: Patient Characteristics and Outcome. Cureus 2019;11:e4113.

2. Schouten LJ, Rutten J, Huveneers HA, et al. Incidence of brain metastases in a cohort of patients with carcinoma of the breast, colon, kidney, and lung and melanoma. Cancer 2002;94:2698-705.

3. Murray N, Sheehan F. Limited stage small cell lung cancer. Curr Treat Options Oncol 2001;2:63-70.

4. Barnholtz-Sloan JS, Sloan AE, Davis FG, et al. Incidence proportions of brain metastases in patients diagnosed (1973 to 2001) in the Metropolitan Detroit Cancer Surveillance System. J Clin Oncol 2004;22:2865-72.

5. Olmez I, Donahue BR, Butler JS, et al. Clinical outcomes in extracranial tumor sites and unusual toxieities with concurrent whole brain radiation (WBRT) and Erlotinib treatment in patients with non-small cell lungcancer (NSCLC) with brain metastasis. Lung Cancer 2010;70:174-9.

6. Preusser M, Capper D, I lhan-Mut lu A, et al. Brain metastases: pathobiology and emerging targeted therapies. Acta Neuropathol 2012;123:205-22.

7. Gaspar L, Scott C, Rotman M, et al. Recursive partitioning analysis (RPA) of prognostic factors in three Radiation Therapy Oncology Group (RTOG) brain metastases trials. Int J Radiat Oncol Biol Phys 1997;37:745-51.

8. Villano JL, Durbin EB, Normandeau C, et al. Incidence of brain metastasis at initial presentation of lung cancer. Neuro Oncol 2015;17:122-8.

9. Yang SC, Kuo CW, Lai WW, et al. Dynamic Changes of Health Utility in Lung Cancer Patients Receiving Different Treatments: A 7-Year Follow-up. J Thorac Oncol 2019. (Epub ahead of print).

10. Sais E, Menéndez JA, Bosch-Barrera J. The practicechanging QUARTZ trial: is there any role for whole brain radiotherapy in patients with non-small cell lung cancer and brain metastases? Transl Cancer Res 2017;6:S201-4.

11. Ainsworth NL, McLean MA, McIntyre DJO, et al. Quantitative and textural analysis of magnetization transfer and diffusion images in the early detection of brain metastases. Magn Reson Med 2017;77:1987-95.

12. Faramand A, Niranjan A, Kano H, et al. Primary or salvage stereotactic radiosurgery for brain metastatic small 
cell lung cancer. J Neurooncol 2019;144:217-25.

13. Lu F, Hou Y, Xia Y, et al. Survival and intracranial control outcomes of whole-brain radiotherapy (WBRT) alone versus WBRT plus a radiotherapy boost in non-smallcell lung cancer with brain metastases: a single-institution retrospective analysis. Cancer Manag Res 2019;11:4255-72.

14. Nardone V, Nanni S, Pastina P, et al. Role of perilesional edema and tumor volume in the prognosis of non-small cell lung cancer (NSCLC) undergoing radiosurgery (SRS) for brain metastases. Strahlenther Onkol 2019;195:734-44.

15. Passiglia F, Rolfo C. Upfront radiation versus EGFRTKI: which is the best approach for EGFR-mutated NSCLC patients with brain metastasis? Transl Cancer Res 2017;6:S533-6.

16. Zustovich F, Ferro A, Lombardi G, et al. BevacizumabBased Therapy for Patients with Brain Metastases from

Cite this article as: Dong K, Liu L, Yu Z, Wu D, Zhang Q, Huang X, Ding J, Song H. Brain metastases from lung cancer with neuropsychiatric symptoms as the first symptoms. Transl Lung Cancer Res 2019;8(5):682-691. doi: 10.21037/ tlcr.2019.10.02
Non-Small-Cell Lung Cancer: Preliminary Results. Chemotherapy 2014;60:294-9.

17. Buss EJ, Wang TJ. Treatment of lung adenocarcinoma brain metastases: what is the role of radiotherapy in the age of precision medicine? Transl Lung Cancer Res 2018;7:S318-20.

18. Gaspar LE, Mehta MP, Patchell RA, et al. The role of whole brain radiation therapy in the management of newly diagnosed brain metastases: a systematic review and evidence-based clinical practice guideline. J Neurooncol 2010;96:17-32.

19. Hart MG, Grant R, Walker M, et al. Surgical resection and whole brain radiation therapy versus whole brain radiation therapy alone for single brain metastases. Cochrane Database Syst Rev 2005;(1):CD003292. 